University of Nebraska - Lincoln

DigitalCommons@University of Nebraska - Lincoln

March 2007

\title{
Dynamic enhancement of the exchange bias training effect
}

\author{
Sarbeswar Sahoo \\ University of Nebraska-Lincoln, sarbeswar@gmail.com \\ Srinivas Polisetty \\ University of Nebraska-Lincoln, polisetty.srinivas@gmail.com \\ Christian Binek \\ University of Nebraska-Lincoln, cbinek@unl.edu \\ Andreas Berger \\ Hitachi Global Storage Technologies, San Jose, California, a.berger@nanogune.eu
}

Follow this and additional works at: https://digitalcommons.unl.edu/physicsbinek

Part of the Physics Commons

Sahoo, Sarbeswar; Polisetty, Srinivas; Binek, Christian; and Berger, Andreas, "Dynamic enhancement of the exchange bias training effect" (2007). Christian Binek Publications. 29.

https://digitalcommons.unl.edu/physicsbinek/29

This Article is brought to you for free and open access by the Research Papers in Physics and Astronomy at DigitalCommons@University of Nebraska - Lincoln. It has been accepted for inclusion in Christian Binek Publications by an authorized administrator of DigitalCommons@University of Nebraska - Lincoln. 


\title{
Dynamic enhancement of the exchange bias training effect
}

\author{
S. Sahoo, S. Polisetty, and Ch. Binek ${ }^{\text {a) }}$ \\ Department of Physics and Astronomy, University of Nebraska, Lincoln, Nebraska 68588-0111 \\ and the Nebraska Center for Materials and Nanoscience, University of Nebraska, Lincoln, Nebraska 68588- \\ 0111
}

\author{
A. Berger \\ San Jose Research Center, Hitachi Global Storage Technologies, San Jose, California 95135
}

(Received 13 November 2006; accepted 4 January 2007; published online 1 March 2007)

\begin{abstract}
Exchange bias in coupled magnetic thin films and its accompanying training effect are fundamental interface phenomena with significant impact in spintronic applications. Both effects are well known in heterosystems of ferro- and antiferromagnetic thin films. Here, we report on the dynamic enhancement of the training effect in an exchange coupled bilayer of soft and hard ferromagnetic materials. Training is referred to as a gradual change of the bias field, which evolves upon cycling the soft layer through consecutive hysteresis loops. Its dynamic enhancement is observed with increasing sweep rate of the applied magnetic field from quasistatic to the fully dynamic range. A dynamically generalized theory based on triggered relaxation is in excellent agreement with the training data. Additionally, we evidence the remarkable universality of our theoretical approach when applying it to the dynamically altered training effect of a conventional exchange bias system involving an antiferromagnetic pinning layer. (C) 2007 American Institute of Physics.
\end{abstract}

[DOI: 10.1063/1.2696989]

\section{INTRODUCTION}

Hysteretic magnetization reversal is an extensively studied nonequilibrium phenomenon with a wide range of applications. In the field of magnetic data storage, for instance, the bistability of the magnetic hysteresis is used for information encoding. In accordance with the nonergodic nature of magnetic hysteresis, dynamic loop broadening ${ }^{1-8}$ or even dynamic phase transitions ${ }^{9-12}$ evolve when the time for sweeping the magnetic hysteresis loop falls below the inherent relaxation time of the magnetization. The latter time scale is determined by domain nucleation and domain wall propagation. The dynamics of the magnetization reversal is typically controlled by various types of defects. On the one hand, defects can pin the domain walls and slow down the domain wall propagation. On the other hand, they act as nucleation centers for magnetic domains in bulk ferromagnets and ferromagnetic (FM) thin films and in turn lower the coercivity.

In addition to the above intrinsic pinning mechanisms, exchange bias (EB) is a well-known extrinsic pinning effect in magnetic thin films. ${ }^{13-18}$ Here, pinning or, equivalently, exchange induced unidirectional anisotropy reflects the magnetic interface coupling between a FM and an adjacent antiferromagnetic (AF) thin film. In this conventional manner, $\mathrm{EB}$ in $\mathrm{AF} / \mathrm{FM}$ heterostructures is initialized by field cooling the heterosystem to below the blocking temperature $T_{B}$, where the AF order establishes at least on mesoscopic scales. ${ }^{19}$ The most striking and widely studied features in EB systems are the shift of the FM hysteresis loop along the magnetic field axis by the amount $\mu_{0} H_{\mathrm{EB}}$ and the EB induced loop broadening. A gradual degradation of the EB field can take place when cycling the heterostructure through consecu-

${ }^{a)}$ Electronic mail: cbinek2@unl.edu tive hysteresis loops. ${ }^{20-24}$ This aging phenomenon is known as a training effect and is quantified by the $\mu_{0} H_{\mathrm{EB}}$ vs $n$ dependence, where $n$ labels the number of loops cycled after initializing the EB via field cooling. EB and the accompanying training effect have been observed in various magnetic systems ranging from core shell magnetic granules ${ }^{25}$ and $\mathrm{AF} / \mathrm{FM}$ thin film heterolayers ${ }^{14,26}$ to intrinsic EB taking place at natural interfaces of ferromagnetic nanodomains embedded in an AF matrix of charge ordered manganites. ${ }^{27}$

\section{EXPERIMENT}

Here, we study the EB training effect in a magnetic bilayer structure ${ }^{28}$ where a $3 \mathrm{~nm}$ thick and magnetically soft $\mathrm{CoCr}$ layer (SL) is an antiferromagnetically exchange coupled via a $0.7 \mathrm{~nm}$ Ru interlayer ${ }^{29}$ with a $15 \mathrm{~nm}$ hard CoPtCrB pinning layer (HL) [see insert of Fig. 1(a)]. The sample was fabricated by sputtering and finds applications in disk recording media. ${ }^{29}$ These all FM exchange bias systems combine FM soft reversal layers and hard FM pinning layers. FM pinning layers provide unique experimental access to the training induced change of the magnetization. They also simplify the theoretical description of the pinning mechanism and reveal in turn the dependence of the bias field on the pinning layer magnetization. ${ }^{28,29}$ The exchange coupled thin film bilayers of soft and hard FM materials show remarkable analogies to conventional AF/FM exchange bias heterostructures. Hence, our experimental results presented here have their correspondence in conventional AF/FM exchange bias systems. We show the universality of our theoretical approach by applying it to both SL/HL and AF/FM exchange coupled systems. In order to stress the formal difference be- 

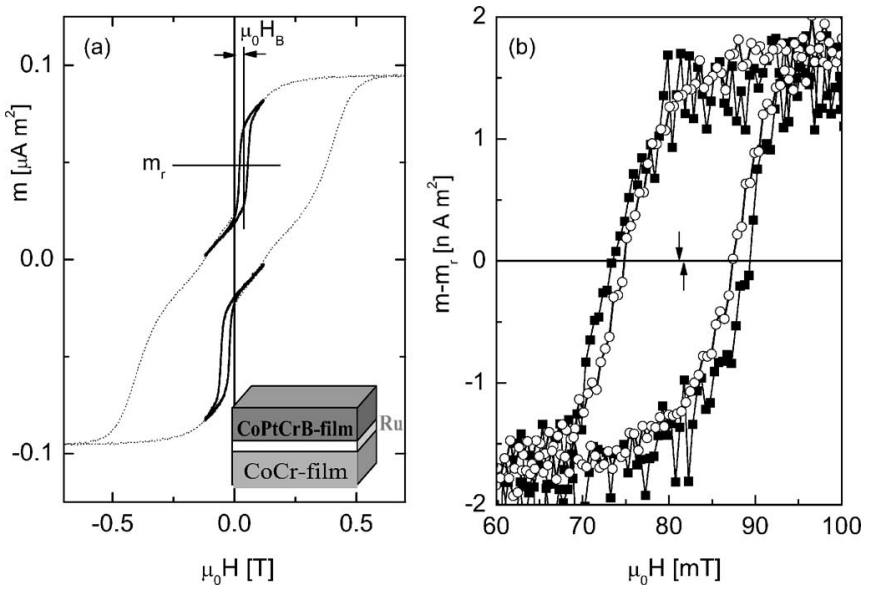

FIG. 1. (a) Overall magnetic hysteresis $m$ vs $\mu_{0} \mathrm{H}$ (dashed line). Thick solid lines are low field minor loops after positive and negative saturation. The horizontal line visualizes $m_{r}$ for the upper SL loop; the vertical line indicates the bias field $\mu_{0} H_{B}$, which is the shift of the SL loop along the field axis relative to $\mu_{0} H=0$. The inset is a schematic view of the sample. (b) Dynamic broadening of the SL loop for sweep rates increasing from $r_{s}$ $=0.2 \mathrm{mT} / \mathrm{s}$ (circles) to $r_{s}=5 \mathrm{mT} / \mathrm{s}$ (squares). The loop broadening is accompanied by a dynamical enhancement of the bias field as indicated by downard $\left(r_{s}=0.2 \mathrm{mT} / \mathrm{s}\right)$ and upward $\left(r_{s}=5 \mathrm{mT} / \mathrm{s}\right)$ pointing arrows.

tween SL/HL and AF/FM heterolayers, we name the shift of the SL loop along the field axis the bias field $\mu_{0} H_{B}$ in contrast to the EB field $\mu_{0} H_{\mathrm{EB}}$.

The experimental study presented here is based on dynamic hysteresis loop measurements on a SL/HL bilayer system. Figure 1(a) displays a schematic structure of the AF coupled FM films and their overall magnetic hysteresis $m$ vs $\mu_{0} H$ (dashed line), where $m$ is the magnetic moment and $H$ is the applied magnetic field. The measurements are done at room temperature by means of alternating gradient force magnetometry (AGFM). The shape of the overall loop reflects the well separated switching field distribution of the HL and SL, respectively. ${ }^{29}$ The thick solid lines are low field minor loops after positive and negative saturation. The low field response of these minor loops is dominated by the magnetization reversal of the soft magnetic $\mathrm{CoCr}$ layer. Their position is shifted along the $m$ axis due to the remanent magnetic moment $m_{r}$ of the $\mathrm{CoPtCrB}$ layer and along the field axis due to the $\mathrm{Ru}$ mediated interlayer exchange coupling.

\section{RESULTS AND ANALYSIS}

Our major objective is to shed light on the intriguing correlation between the dynamics of the SL magnetization reversal and the training of the bias field. More specifically, we show that the $\mu_{0} H_{B}$ vs $n$ dependence of the SL bias field depends on the sweep rate $r_{s}=d \mu_{0} H / d t$ of the SL hysteresis loops. In addition, we show that the value of the equilibrium bias field, $\mu_{0} H_{B}^{e}=\mu_{0} H_{B}(n \rightarrow \infty)$, reflects the dynamic broadening of the SL hysteresis and follows a power law behavior with respect to the sweep rate. The dynamically altered training effect is quantitatively modeled when generalizing our recent theoretical approach from a quasistatic description into a dynamic form. ${ }^{28,30,31}$ This dynamic generalization is motivated by the competition between a finite sweep rate of the SL hysteresis and triggered but noninstantaneous relax-

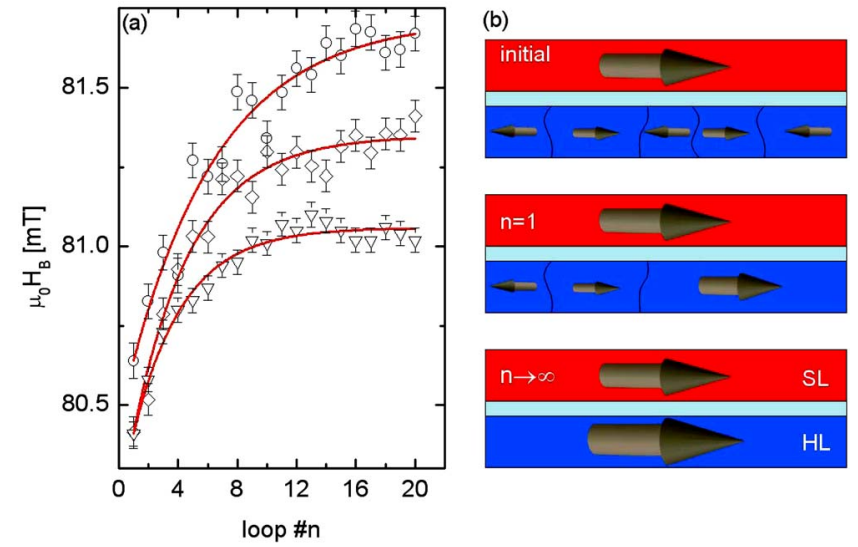

FIG. 2. (Color online) (a) Training effect $\mu_{0} H_{B}$ vs $n$ of the HL-SL bilayer for various sweep rates $r_{s}=5 \mathrm{mT} / \mathrm{s}$ (circles), $1.67 \mathrm{mT} / \mathrm{s}$ (rhombohedra), and $1.0 \mathrm{mT} / \mathrm{s}$ (down triangles). The bars reflect the errors in deducing the bias fields from hysteresis loops. The initial magnetization state of the HL is set prior to each training sequence by a set field of $\mu_{0} H_{\text {set }}=-0.31 \mathrm{~T}$ after saturation in $\mu_{0} H=0.8 \mathrm{~T}$. Lines are best fits of Eq. (5) to the data. (b) Sketch illustrating the training effect. Figures represent the SL (top) HL (bottom) bilayer exchange coupled via the $\mathrm{Ru}$ interlayer in their respective states of magnetization. Arrows indicate direction and strength of the magnetization. Initially, the HL breaks into magnetic domains. The first SL loop $(n=1)$ gives rise to the rearrangement of the HL spin structure. The successively increasing $\mathrm{HL}$ magnetization reaches the quasiequilibrium value in the limit $n \rightarrow \infty$.

ation of the pinning layer magnetization during the SL loop. The latter relaxation process is truncated if the field sweep controlling the SL loop is fast in comparison with the free HL relaxation.

Figure 1(b) shows the dynamic broadening of the SL hysteresis for $n=20$ when the sweep rate is increased from $r_{s}=0.2 \mathrm{mT} / \mathrm{s}$ (circles) to $r_{s}=5 \mathrm{mT} / \mathrm{s}$ (squares). The upwards and down pointing arrows mark the bias fields for $r_{s}$ $=5 \mathrm{mT} / \mathrm{s}$ and $r_{s}=0.2 \mathrm{mT} / \mathrm{s}$, respectively, indicating the correlation between dynamic loop broadening and the increase of the bias field. The associated noise level increases with increasing sweep rate in accordance with a decrease of the instrumental integration time. Note that corresponding reference measurements with a standard $\mathrm{Ni}$ gauge sample show no sweep rate dependent broadening of the hysteresis. Therefore, we evidence that the observed dynamic broadening originates from intrinsic physics of the bilayer system.

Figure 2(a) shows the training effect of the FM bilayer for various sweep rates $r_{s}=5 \mathrm{mT} / \mathrm{s}$ (circles), $1.67 \mathrm{mT} / \mathrm{s}$ (rhombohedra), and $1.0 \mathrm{mT} / \mathrm{s}$ (down triangles). Each $\mu_{0} H_{B}$ vs $n$ data set shows the bias fields of 20 subsequently measured minor hysteresis loops of the SL. These minor loops are measured in the field interval $0 \leqslant \mu_{0} H \leqslant 140 \mathrm{mT}$, which do not switch the HL. The data sets are taken after saturating the bilayer in $\mu_{0} H=0.8 \mathrm{~T}$ and subsequent partial demagnetization of the pinning layer in a static set field of $\mu_{0} H_{\mathrm{set}}=$ $-0.31 \mathrm{~T}$. The data clearly show that the strength of the training effect increases with increasing sweep rate, while at the same time this increase in relaxation is spread out over more SL hysteresis loop cycles. Our subsequently outlined theoretical approach sheds light on this phenomenon.

Training in SL/HL bilayers as well as AF/FM heterostructures is successfully described in the framework of spin 
configurational relaxation of the magnetic pinning layer. The relaxation process is activated through each of the consecutively cycled hysteresis loops within a training sequence. Figure 2(b) displays a cartoon visualizing the process of triggered relaxation and training. After applying the set field $\mu_{0} H_{\text {set }}=-0.31 \mathrm{~T}$ the $\mathrm{HL}$ is in its initial domain state of reduced magnetization. The first SL hysteresis triggers partial relaxation of the HL towards its equilibrium of increased magnetization. This process continues with subsequent cycles until the HL reaches in the limit $n \rightarrow \infty$ its magnetization state of quasiequilibrium. It is worth mentioning that training reflects the tendency of the pinning layer to approach its equilibrium spin configuration. This holds for all FM systems where the bias field can increase as well as for AF pinning layers where the exchange bias field always decreases through consecutive training cycles $n$. The equilibrium spin configuration is given by a positively (increasing $\mu_{0} H_{B}$ vs $n$ ) or negatively (decreasing $\mu_{0} H_{B}$ vs $n$ ) magnetized state in the case of a FM pinning layer but is a demagnetized state in the case of an $\mathrm{AF}$ pinning layer.

Recently, we showed that this triggered process is adequately described when discretizing the dynamic LandauKhalatnikov (LK) equation ${ }^{28,30,31}$

$$
\xi \dot{S}=-\frac{\partial \Delta F}{\partial S},
$$

where $\xi$ is a phenomenological damping constant and $\Delta F$ $=a\left(S-S_{e}\right)^{2}+O\left(\delta S^{4}\right)$ is the first order approximation of the nonequilibrium free energy in the case of a FM pinning layer. $\Delta F$ quantifies the increase of the free energy when the interface magnetization $S$ of the pinning layer deviates from its quasiequilibrium value, $S_{e}$. The latter determines the bias field in the limit $n \rightarrow \infty$ and depends on the initial state of the pinning layer and the sweep rate. Discretization of Eq. (1) is achieved by replacing the continuous temporal change of the interface magnetization $\dot{S}$ with a temporal average according to

$$
\dot{S} \rightarrow \frac{1}{\widetilde{\tau}} \int_{t_{n}}^{t_{n}+\tilde{\tau}} \dot{S} d t=\frac{S\left(t_{n}+\widetilde{\tau}\right)-S\left(t_{n}\right)}{\widetilde{\tau}},
$$

where

$$
\tilde{\tau}= \begin{cases}\tau & \text { if } \Delta t \gg \tau \\ \Delta t & \text { if } \Delta t \ll \tau .\end{cases}
$$

Here, $\Delta t=2 \mu_{0} \Delta H_{\max } / r_{s}$ is the experimental time interval required for the measurement of a hysteresis loop in the field range $0 \leqslant \mu_{0} H \leqslant \mu_{0} \Delta H_{\max }$, where $\mu_{0} \Delta H_{\max }=140 \mathrm{mT}$. $\tau$ is the intrinsic time scale for which nontruncated relaxation of the pinning layer magnetization takes place. The time interval between two subsequent loops is virtually free from relaxation and is, hence, not involved in Eq. (2). Note that the experimental finding of an increasing training effect with increasing sweep rate confirms that training does not depend on the time the pinning layer (HL) is exposed to the external magnetic field. This exposure time actually decreases with increasing $r_{s}$. Instead, training is a discontinuous process triggered by the SL hysteresis loop while relaxation is absent otherwise. Recently, we found independent evidence of this fact when studying the correlation between the onset of SL hysteresis and the training effect in the quasistatic limit of low sweep rates. ${ }^{28}$

In the quasistatic regime the experimental time interval of an individual hysteresis loop is large enough to allow for triggered but subsequently unperturbed relaxation of $S$. This free relaxation of the pinning layer magnetization takes place on the characteristic time scale $\tau$ such that $\widetilde{\tau} \rightarrow \tau$. In the limit of fast field sweeps when $\Delta t$ becomes smaller than $\tau$, truncation of the relaxation process sets in which is expressed by $\widetilde{\tau} \rightarrow \Delta t$. The formal description of the crossover from truncated to free relaxation of the pinning layer can be considered in the framework of a mechanical analog of an overcritically damped oscillator. The effect of the SL hysteresis loop on the HL magnetization relaxation resembles a time dependent external driving force in this mechanical picture. The general solution of the differential equation of a damped driven oscillator requires the superposition of a transient and a stationary component. The transient exponentially decaying solution corresponds to the free relaxation of the HL which dominates the temporal evolution when the stationary component is virtually constant. This is the quasi static limit of slowly varying forces or low sweep rates such that $\Delta t$ $\gg \tau$. In the case of high sweep rates or fast varying forces the transient solution is virtually constant on the time scale $\Delta t$, where the external force significantly changes. Since the weighting of the transient contribution decays exponentially for $\Delta t \rightarrow 0$ or high sweep rates, the crossover from $\widetilde{\tau} \approx \tau$ to $\widetilde{\tau} \approx \Delta t$ is given by

$$
\tilde{\tau}=\tau\left(1-e^{-\Delta t / \tau}\right),
$$

where $\Delta t=2 \mu_{0} \Delta H_{\max } / r_{s}$. The crossover expression (3) reflects the key element of the dynamic generalization of our former quasistatic approach outlined in Ref. 28. Replacing the characteristic time constant $\tau$ by the dynamically generalized $\tilde{\tau}$ of Eq. (4) throughout the derivation of $\mu_{0} H_{B}$ $=\mu_{0} H_{B}(n)$ yields

$$
\begin{aligned}
\mu_{0} H_{B}(n)= & (\tilde{K}+1)^{n-1}\left\{\mu_{0} H_{B}(n=1)\right. \\
& \left.-\tilde{K} \mu_{0} H_{B}^{e}\left[\frac{(\widetilde{K}+1)^{n+1}-1}{\widetilde{K}(\tilde{K}+1)^{n-1}}-(\tilde{K}+2)\right]\right\} .
\end{aligned}
$$

Equation (5) is identical to the quasistatic expression derived in Ref. 28 when replacing the constant $K \propto \tau$ by $\widetilde{K} \propto \widetilde{\tau}$. Lines in Fig. 2 show the best fits of Eq. (5) to the $\mu_{0} H_{B}$ vs $n$ data using $\widetilde{K}$ and $\mu_{0} H_{B}^{e}$ as fitting parameters. Note that the numerical values for $\tilde{K}$ need to be limited to $-1<\tilde{K}<0$ in order to facilitate convergence.

Figures 3(a) and 3(b) display the sweep rate dependence of the absolute value of the resulting fitting parameters $|\tilde{K}|$ vs $r_{s}$ and $\mu_{0} H_{B}^{e}$ vs $r_{s}$, respectively. In accordance with Eq. (4) the line in Fig. 3(a) is the best fit of $|\widetilde{K}|=c_{1}\left(1-e^{-c_{2} / r_{s}}\right)$, where the fitting parameter $c_{1}=0.244 \pm 0.003$ takes into account the proportionality between $|\tilde{K}|$ and $\tilde{\tau}$ while $c_{2}=4.3 \pm 0.3 \mathrm{mT} / \mathrm{s}$ accounts for the proportionality between $\Delta t$ and the inverse 

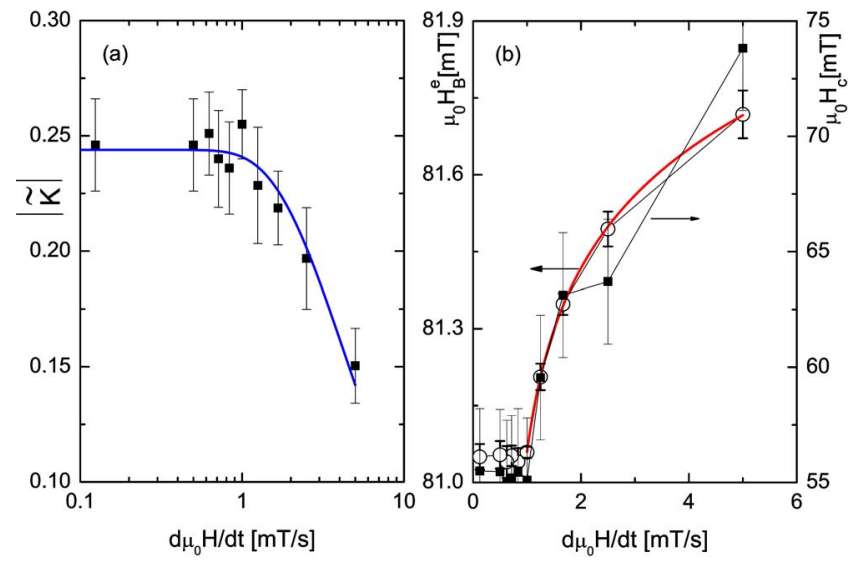

FIG. 3. (Color online) (a) Sweep rate dependence of the fit parameter $\tilde{K}$ resulting from the fits shown in Fig. 2(a) and additional data sets (not shown). The bars reflect the errors resulting from a best fit using the Levenberg-Marquardt algorithm. The $|\tilde{K}|$ vs $r_{s}$ data are in turn fitted to $|\tilde{K}|$ $=c_{1}\left(1-e^{-c_{2} / r_{s}}\right)$ in accordance with Eq. (4). The line is the result of the best fit. (b) Sweep rate dependence of the fit parameter $\mu_{0} H_{B}^{e}$ (circles, left frame) and the SL loop width $\mu_{0} H_{c}=\mu_{0}\left(H_{c_{2}}-H_{c_{1}}\right) / 2$ (squares, right frame). The line is a best fit of the empirical power law $\mu_{0} H_{B}^{e}=A\left[r_{s}-r_{s}(1 \mathrm{mT} / \mathrm{s})\right]^{\eta}$, with $\eta=3 \times 10^{-3}$.

sweep rate. The two-parameter fit is in excellent agreement with the data confirming the overall approach and its dynamic generalization.

Figure 3(b) (circles, left frame) shows the sweep rate dependence of the fitting parameter $\mu_{0} H_{B}^{e}$ obtained from best fits of Eq. (5) to the training data [see Fig. 2(a)]. The same figure (right frame, squares) shows the sweep rate dependence of the coercivity $\mu_{0} H_{c}$, where $\mu_{0} H_{c}=\mu_{0}\left(H_{c_{2}}-H_{c_{1}}\right) / 2$ is the width of the SL loop determined by the intercepts $\mu_{0} H_{c_{1}, c_{2}}$ of the loop $m-m_{r}$ vs $\mu_{0} H$ with the field axis [see Fig. 1(b)]. Clearly, there is a strong correlation between the bias field and the loop width. This type of correlation is a well-known phenomenon in EB systems. ${ }^{7,32,33}$ The origin of the sweep rate dependence of $\mu_{0} H_{B}^{e}$ is therefore reduced to the dynamic broadening of the SL loop. The solid line in Fig. 3(b) represents an empirical power law fit suggested for AF/FM EB systems in Ref. 6. The plateaulike behavior of $\mu_{0} H_{B}^{e}$ vs $r_{s}$ for $r_{s}<1 \mathrm{mT} / \mathrm{s}$ [see Fig. 3(b)] corresponds to the asymptotic approach $\left|\tilde{K}\left(r_{s}<1 \mathrm{mT} / \mathrm{s}\right)\right| \rightarrow 0.244$ [see Fig. 3(a)] indicating the onset of quasistatic behavior consistent throughout both data sets. Note that Figs. 3(a) and 3(b) show data points obtained from the fits displayed in Fig. 2(a) and additional fitting results not explicitly shown.

Recently, the sweep rate dependence of the EB training has been studied in the AF/FM heterosystem $\mathrm{Ni}_{81} \mathrm{Fe}_{19}$ $(6 \mathrm{~nm}) / \mathrm{Ir}_{22} \mathrm{Mn}_{78}(2 \mathrm{~nm})$ (Ref. 6). By reanalyzing the data presented in Ref. 6 we evidence here the universality of our approach and show that the hitherto used power law description of the $\mu_{0} H_{\mathrm{EB}}$ vs $n$ dependence has only some meaning in the limit of large $n$ but generically fails in the vicinity $n$ $\rightarrow 1$. Moreover, we show that the sweep rate dependence of the fitting parameters is consistent with the process of truncation quantified in Eq. (4).

Open symbols in Fig. 4 show the experimental results obtained by Heiwan et al. for sweep rates $r_{s}=10 \mathrm{mT} / \mathrm{s}$ (rhombohedra), $r_{s}=50 \mathrm{mT} / \mathrm{s}$ (squares), and $r_{s}=100 \mathrm{mT} / \mathrm{s}$

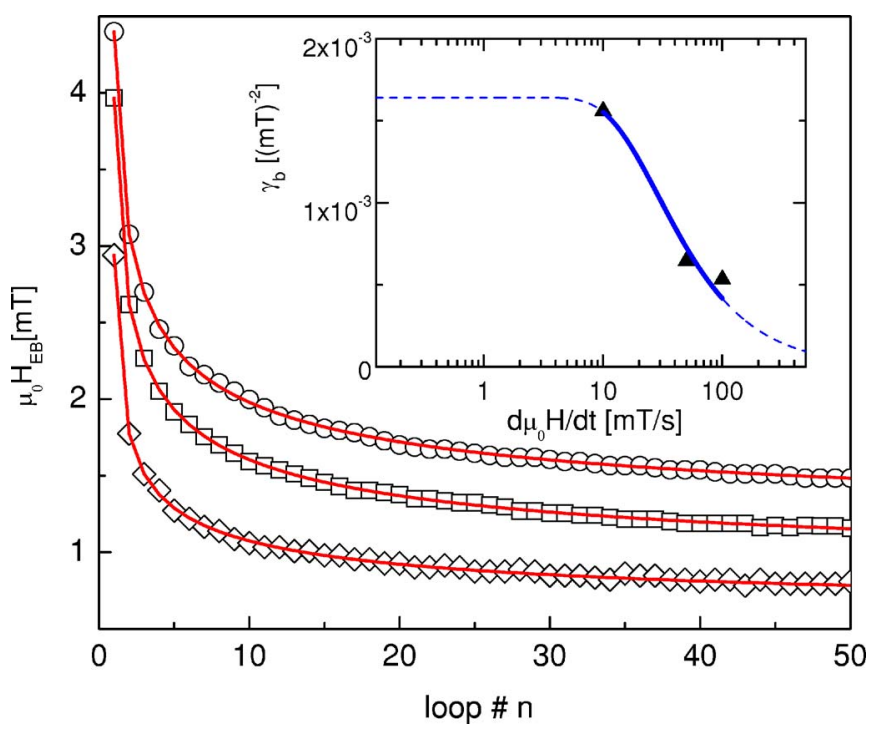

FIG. 4. (Color online) Training $\mu_{0} H_{\mathrm{EB}}$ vs $n$ of conventional AF/FM exchange bias heterostructures for various sweep rates $r_{s}=10 \mathrm{mT} / \mathrm{s}$ (rhombohedra), $50 \mathrm{mT} / \mathrm{s}$ (squares), and $100 \mathrm{mT} / \mathrm{s}$ (circles). Data (open symbols) are taken from Ref. 6. Lines are the best fits of the sequence (6). Note that the lines have physical meaning only at integer values. The inset shows the sweep rate dependence of the fitting parameter $\gamma_{b}$. The solid line in turn is the best fit of $\gamma_{b}=c_{b}\left(1-e^{-c_{b} / r_{s}}\right)$ to the data $\gamma_{b}$ vs $r_{s}$. The dashed line is an extrapolation of the fit.

(circles). Lines represent the best fits of our theoretical approach based on the discretized LK equation [Eq. (1)]. Here the structure of the free energy has been adapted to the AF spin ordering of the pinning layer. ${ }^{30,31}$ In addition to our basic approach outlined in Refs. 30 and 31, we extended here the free energy expansion with a correction of the leading term. By doing so we achieve virtually perfect fitting results (see lines in Fig. 4) for $1 \leqslant n \leqslant 50$ from

$$
\begin{aligned}
\mu_{0}\left[H_{\mathrm{EB}}(n+1)-H_{\mathrm{EB}}(n)\right]= & -\gamma_{b}\left\{\mu_{0}\left[H_{\mathrm{EB}}(n)-H_{\mathrm{EB}}^{e}\right]\right\}^{3} \\
& -\gamma_{c}\left\{\mu_{0}\left[H_{\mathrm{EB}}(n)-H_{\mathrm{EB}}^{e}\right]\right\}^{5},
\end{aligned}
$$

where $\mu_{0} H_{\mathrm{EB}}^{e}$ is the fitting parameter describing $\mu_{0} H_{\mathrm{EB}}$ vs $n$ in the limit $n \rightarrow \infty$. The new parameter $\gamma_{c}$ results from the higher order expansion of the free energy and hence $\left|\gamma_{c}\right|$ $\ll\left|\gamma_{b}\right|$. Both $\gamma_{b}$ and $\gamma_{c}$ are proportional to $\widetilde{\tau}=\widetilde{\tau}\left(r_{s}\right)$ giving rise to their respective sweep rate dependence in accordance with Eq. (4). The implicit sequence (6) is a straightforward refinement of our recent approach outlined in Refs. 30 and 31 and evidenced for diverse exchange coupled systems. ${ }^{27,28,30,31,34}$ Note that Eq. (6) involves three fitting parameters. The identical number of free parameters is required for the power law description first introduced in Ref. 20. However, the physically motivated Eq. (6) provides far better fitting results for all sweep rates and explains in a consistent manner the $r_{s}$ dependence of the fitting parameters. The inset of Fig. 4 shows $\gamma_{b}$ vs $r_{s}$. The solid line is the best fit of Eq. (4) to the data points. The dashed line displays the extrapolated result of the fit towards the quasistatic as well as the high sweep rate regime. The result is in accordance with the predicted exponential behavior of $\tilde{\tau}=\tilde{\tau}\left(r_{s}\right)$. 


\section{CONCLUSIONS}

The present results evidence a dynamic enhancement of the exchange bias and its training effect in conventional and in our all ferromagnetic bilayer structures. Exchange coupled films based on all ferromagnetic materials provide unique access to the magnetization state of the pinning layer by means of simple magnetometry. The bias field which quantifies the shift of the hysteresis loop of the soft ferromagnetic layer along the field axis increases with increasing sweep rate of the magnetic field. The increase of the bias field is correlated with a dynamic broadening of the soft layer hysteresis. Both the bias field increase and the SL loop broadening follow the same power law behavior above a quasistatic threshold. The training, which is the dependence of the bias field on the number of subsequently cycled loops, is furthermore dynamically spread out. This effect is described here in the framework of a dynamically generalized theoretical approach, which is in excellent agreement with our experimental data. The analysis of the sweep rate dependence of the fitting parameters evidences in turn the key element of the dynamically generalized theory which is based on truncated relaxation of the pinning layer magnetization. We also demonstrate that the same generalization which models the dynamic enhancement of the training effect in ferromagnetic bilayers applies to conventional exchange bias systems when the free energy is adapted to the antiferromagnetic order of the pinning layer. These studies have important implications for concepts of data storage. ${ }^{25}$ It has to be shown, for instance, that the recently observed enhancement of the blocking temperature of magnetic nanoparticles via exchange bias remains dynamically stable.

\section{ACKNOWLEDGMENTS}

Work at the University of Nebraska-Lincoln is supported by NSF through Career DMR-0547887 and by NRI.
${ }^{1}$ B. Raquet, R. Mamy, and J. C. Ousset, Phys. Rev. B 54, 4128 (1996).

${ }^{2}$ J.-S. Suen and J. L. Erskine, Phys. Rev. Lett. 78, 3567 (1997).

${ }^{3}$ R. L. Stamps, Phys. Rev. B 61, 12174 (2000).

${ }^{4}$ Z. Yang et al., J. Appl. Phys. 87, 5729 (2001).

5 J. Camarero et al., Phys. Rev. B 64, 172402 (2001).

${ }^{6}$ H. Xi, R. M. White, S. Mao, Z. Gao, Z. Yang, and E. Murdock, Phys. Rev. B 64, 184416 (2001)

${ }^{7}$ Y. Pennec et al., Phys. Rev. B 69, 180402 (2004).

${ }^{8}$ T. A. Moore, M. J. Walker, A. S. Middleton, and J. A. C. Bland, J. Appl. Phys. 97, 053903 (2005).

${ }^{9}$ B. K. Chakrabarti and M. Acharyya, Rev. Mod. Phys. 71, 847 (1999).

${ }^{10}$ T. Nattermann, V. Pokrovsky, and V. M. Vinokur, Phys. Rev. Lett. 87, 197005 (2001).

${ }^{11}$ X. Chen, O. Sichelschmidt, W. Kleemann, O. Petracic, Ch. Binek, J. B. Sousa, S. Cardoso, and P. P. Freitas, Phys. Rev. Lett. 89, 137203 (2003).

${ }^{12}$ S. W. Sides, P. A. Rikvold, and M. A. Novotny, Phys. Rev. Lett. 81, 834 (1998).

${ }^{13}$ W. H. Meiklejohn and C. P. Bean, Phys. Rev. 105, 904 (1957).

${ }^{14}$ J. Nogués and I. K. Schuller, J. Magn. Magn. Mater. 192, 203 (1999).

${ }^{15}$ A. Berkowitz and K. Takano, J. Magn. Magn. Mater. 200, 552 (1999).

${ }^{16}$ R. L. Stamps, J. Phys. D 33, R247 (2000).

${ }^{17}$ M. Kiwi, J. Magn. Magn. Mater. 234, 584 (2001).

${ }^{18}$ W. Kuch, L. I. Chelaru, F. Offi, J. Wang, M. Kotsugi, and J. Kirschner, Nat. Mater. 5, 128 (2006).

${ }^{19}$ I. Roshchin, O. Petracic, R. Morales, Z.-P. Li, X. Batlle, and I. K. Schuller, Europhys. Lett. 71, 297 (2005).

${ }^{20}$ D. Paccard, C. Schlenker, O. Massenet, R. Montmory, and A. Yelon, Phys. Status Solidi 16, 301 (1966).

${ }^{21}$ C. Schlenker, S. S. P. Parkin, J. C. Scott, and K. Howard, J. Magn. Magn. Mater. 54, 801 (1986).

${ }^{22}$ K. Zhang, T. Zhao, and M. Fujiwara, J. Appl. Phys. 89, 6910 (2001).

${ }^{23}$ S. G. te Velthuis, A. Berger, G. P. Felcher, B. Hill, and E. Dahlberg, J. Appl. Phys. 87, 5046 (2000).

${ }^{24}$ A. Hoffmann, Phys. Rev. Lett. 93, 097203 (2004).

${ }^{25}$ V. Skumryev, S. Stoyanov, Y. Zhang, G. Hadjipanayis, D. Givord, and J. Nogués, Nature (London) 423, 850 (2003).

${ }^{26}$ A. Hochstrat, Ch. Binek, and W. Kleemann, Phys. Rev. B 66, 092409 (2002).

${ }^{27}$ D. Niebieskikwiat and M. B. Salamon, Phys. Rev. B 72, 174422 (2005).

${ }^{28}$ Ch. Binek, S. Polisetty, Xi. He, and A. Berger, Phys. Rev. Lett. 96, 067201 (2006).

${ }^{29}$ A. Berger, D. T. Margulies, and H. Do, Appl. Phys. Lett. 85, 1571 (2004). ${ }^{30}$ Ch. Binek, Phys. Rev. B 70, 014421 (2004).

${ }^{31} \mathrm{Ch}$. Binek, X. He, and S. Polisetty, Phys. Rev. B 72, 054408 (2005).

${ }^{32}$ M. Ali, C. H. Marrows, and B. J. Hickey, Phys. Rev. B 67, 172405 (2003).

${ }^{33}$ J. Sort, B. Dieny, and J. Nogués, Phys. Rev. B 72, 104412 (2005).

${ }^{34}$ Y.-K. Tang, S. Young, and Z.-H. Cheng, Phys. Rev. B 73, 174419 (2006). 